\title{
SECOND ORDER ELLIPTIC EQUATIONS WITH DEGENERATE WEIGHT
}

\author{
W. ALLEGRETTO
}

(Communicated by Barbara L. Keyfitz)

\begin{abstract}
We consider the eigenvalue problem: $-\Delta u-q u=\lambda \omega u, u \in$ $\stackrel{\circ}{H}^{1,2}(\Omega)$, in a smooth bounded domain $\Omega \subset \mathbf{R}^{n}$. We allow $-\Delta-q$ to have negative spectrum and assume $\omega \geq 0$ in $\Omega, \omega \equiv 0$ in a subdomain of $\Omega$. Under suitable regularity conditions, we establish several results for the spectrum of this problem. In particular, we give: a min.max. formula for $\lambda$; a precise estimate on the number of negative $\lambda$; an estimate for the location of negative $\lambda$. An example concludes the paper.
\end{abstract}

\section{INTRODUCTION}

We consider in this paper the eigenvalue problem:

$$
\ell u=-\Delta u-q u=\lambda \omega u
$$

in a smooth bounded domain $\Omega \subset \mathbf{R}^{n}$ with $u \in \stackrel{\circ}{H}^{1,2}(\Omega)$ and $q, \omega \in L^{\infty}(\Omega)$. The spectral theory of (1) is well known if either $\ell$ or $\omega$ is positive, (i.e. $(\ell \varphi, \varphi)>0,(\omega \varphi, \varphi)>0$ for $\left.\varphi \in C_{0}^{\infty}(\Omega), \varphi \not \equiv 0\right)$, see e.g. [4], [9]. The situation is considerably different if neither $\ell$ nor $\omega$ is assumed positive. For example, it is possible for (1) to have complex eigenvalues in such a situation, see e.g. [1], [2], despite the symmetrical appearance of the problem. We consider here a problem which is intermediate between the cases mentioned above. Specifically, while we allow the existence of $\varphi \in C_{0}^{\infty}(\Omega)$ such that $(\ell \varphi, \varphi)<0$, we also postulate the existence of a nonempty subdomain $\Omega^{\prime} \Subset \Omega$ such that $\Omega-\bar{\Omega}^{\prime}=\Omega^{\prime \prime}$ with $\Omega^{\prime \prime}$ also a domain and $\omega>0$ a.e. $\Omega^{\prime}, \omega=0$ a.e. $\Omega^{\prime \prime}$. For convenience, we assume throughout that $\partial \Omega^{\prime} \in C^{\infty}$. Much of the motivation for our work comes from two papers of Everitt, Kwong and Zettl, [5], [6] where the $n=1$ case of this problem was considered (under much more general conditions on the coefficients and the boundary conditions), and where more references may be found. It seems unreasonable to expect that our methods will

Received by the editors August 30, 1988 and, in revised form, January 13, 1989.

1980 Mathematics Subject Classification (1985 Revision). Primary 35P15, 35J20, 35B05.

Research sponsored by NSERC. 
lead to new results when specialized to ordinary differential equations. Nevertheless, we obtain some modest new results even for this case as we indicate below.

Briefly, our results are as follows: We first show that (1) has infinitely many real eigenvalues. We next note that the standard Courant min.max. principle may not be valid, and give a suitable modification. Finally, we give an exact formula for the number of negative eigenvalues of (1) and show that this number depends on $\Omega, \Omega^{\prime}, \Omega^{\prime \prime}$ but not on the specific values of $\omega$ on $\Omega^{\prime}$. We remark that simple two-parameter arguments are very useful in these considerations, and we recall the important results of Binding and Browne on this subject, see e.g. [3] and the references therein. Next, we apply these results to estimate the location of the negative spectrum, $S^{-}$, of $\ell$ (if any). As may be expected, our estimates depend strongly on the values of $\omega$ in $\Omega^{\prime}$. We end the paper with an illustrative example.

In conclusion, we remark that exactly the same proofs hold with very little or no change for more general uniformly elliptic expressions in place of $-\Delta$. In the same way, we could consider more general boundary conditions by assuming $u \in V$, with $\stackrel{\circ}{H}^{1,2}(\Omega) \subset V \subset H^{1,2}(\Omega)$, in place of $u \in \stackrel{\circ}{H}^{1,2}(\Omega)$. In this manner, we can consider some problems corresponding to mixed Dirichlet-Neumann boundary conditions. Unique continuation, however, seems to be an essential requirement in our proofs.

\section{RESULtS}

To deal with (1) we introduce the following eigenvalue problems:

$$
\begin{cases}\ell u-\lambda \omega u=k_{j}(\lambda) u, & \\ u \in \stackrel{\circ}{H} & \stackrel{\circ}{H}{ }^{1,2}(\Omega) .\end{cases}
$$

$$
\begin{aligned}
& \left\{\begin{array}{l}
\ell v=\mu v, \\
v \in \stackrel{\circ}{H}^{1,2}(\Omega) .
\end{array}\right. \\
& \left\{\begin{array}{l}
\ell z=\delta z, \\
z \in \stackrel{\circ}{H}^{1,2}\left(\Omega^{\prime \prime}\right) .
\end{array}\right.
\end{aligned}
$$

Observe that $\left(1^{\prime}\right)$ is an embedding of our problem in a family of two-parameter problems. Note that: $k_{j}(\lambda)$ is given by the Courant min.max., [10, p. 75], in the usual way; the eigenvalues of $(1)$ are $\bigcup_{j} k_{j}^{-1}(0)$; the weight function $\omega$ does not appear in (2), (3).

We begin with:

\section{Theorem 1.}

(a) Let $u \in \stackrel{\circ}{H}^{1,2}(\Omega), u=0$ a.e. $\Omega^{\prime}$. Then $u \in \stackrel{\circ}{H}^{1,2}\left(\Omega^{\prime \prime}\right)$.

(b) $k_{j} \in H_{\mathrm{loc}}^{1, \infty}\left(\mathbf{R}^{1}\right)$ and $k_{j}^{\prime}<0$ a.e. 
(c) $\lim _{\lambda \rightarrow-\infty} k_{j}(\lambda)=\delta_{j}, j=1, \ldots$

(d) $k_{j}(0)=\mu_{j}, j=1, \ldots$.

(e) Equation (1) has infinitely many eigenvalues.

(f) Let $\left\{u_{j}\right\}_{j=1}^{p}$ be eigenfunctions of $\left(1^{\prime}\right)$ and assume $\sum_{j=1}^{p} c_{j} u_{j}=0$ a.e. in $\Omega^{\prime}$. Then $\sum_{j=1}^{p} c_{j} u_{j}=0$ a.e. $\Omega$.

Proof. (a) Let $u \in \stackrel{\circ}{H}^{1,2}(\Omega)$ and $u=0$ in $\Omega^{\prime}$. Construct a properly nested family of smooth domains $\Omega_{i}$ such that

$$
\Omega^{\prime}=\bigcup_{1}^{\infty} \Omega_{i}, \quad \text { and observe } \overline{\Omega^{\prime \prime}} \cap \Omega=\bigcap_{i=1}^{\infty}\left(\Omega-\bar{\Omega}_{i}\right) .
$$

An elementary mollification argument shows that $u \in \stackrel{\circ}{H}^{1,2}\left(\Omega-\bar{\Omega}_{i}\right)$ for any $i$, whence $u \in \stackrel{\circ}{H}^{1,2}\left(\operatorname{int} \bigcap_{i=1}^{\infty}\left(\Omega-\bar{\Omega}_{i}\right)\right)=\stackrel{\circ}{H}^{1,2}\left(\Omega^{\prime \prime}\right)$ as a consequence of the smoothness of $\partial \Omega^{\prime}$ (see [13], and also [14]). In one dimension this result is immediate, since $u$ is continuous.

(b) The Courant min.max. principle implies that $\left|k_{j}(\lambda)-k_{j}(\mu)\right| \leq\|\omega\|_{L^{\infty}} \times$ $|\lambda-\mu|$. We need only to apply Rademaker's theorem, [7, p. 174, Prob. 7], to show that $k_{j} \in H_{\text {loc }}^{1, \infty}\left(\mathbf{R}^{1}\right)$. Finally observe that if $k_{j}(\lambda)$ has multiplicity $p$, i.e. $k_{j-\alpha}(\lambda)=k_{j+\beta}(\lambda) \triangleq k$ with $\alpha, \beta \geq 0, \alpha+\beta=p-1$, then for $|h|$ sufficiently small we may construct eigenvalues $\tau_{i}(\lambda+h)$ and associated eigenvectors $\varphi_{i}(\lambda+h)$ as power series in $h$ for $i=j-\alpha, \ldots, j+\beta$, see e.g. $\left[11\right.$, p. 74]. Observe that since $\left\{\tau_{i}\right\}_{i=j-\alpha}^{j+\beta}$ are power series, we may order this set for $h>0$ sufficiently small and, without loss of generality assume, $\tau_{i} \leq \tau_{k}$ for $i \leq k$. We then select the eigenvector associated with $k_{j}(\lambda+h)$ to be $\varphi_{j}(\lambda+h)$ for $h \geq 0$, small. Set $v_{m}=\varphi_{j}\left(\mu_{m}\right), u=\varphi_{j}(\lambda)$ and suppose now $k_{j}^{\prime}(\lambda)$ exists. Then, taking inner products, we observe

$$
\begin{aligned}
k_{j}^{\prime}(\lambda) & =\lim _{\mu_{n} \rightarrow \lambda^{+}}\left[\frac{k_{j}\left(\mu_{n}\right)-k_{j}(\lambda)}{\mu_{n}-\lambda}\right]=\lim _{\mu_{n} \rightarrow \lambda^{+}}\left(-\omega u, v_{n}\right) \\
& =-(\omega u, u) \leq 0 .
\end{aligned}
$$

Finally, suppose $(\omega u, u)=0$. Then $u=0$ a.e. on $\operatorname{supp}(\omega)$. The Unique Continuation Theorem given in [10] shows $u=0$ a.e. $\Omega$, which is a contradiction. We conclude $k_{j}^{\prime}<0$ a.e.

(c) We first show that this holds for $j=1$. The general result will then follow by induction. Observe first that $\delta_{1} \geq k_{1}(\lambda)$ for any $\lambda$ by the Courant min.max., and consequently, $\delta_{1} \geq \delta \triangleq \lim _{\lambda \rightarrow-\infty} k_{1}(\lambda)$ by Part (b). Now let $\left\{u_{i}\right\}$ satisfy

$$
\ell u_{i}-\lambda_{i} \omega u_{i}=k_{1}\left(\lambda_{i}\right) u_{i}
$$

with $\lambda_{i} \rightarrow-\infty$, and $\left(u_{i}, u_{i}\right)=1$. We observe that $\left(\ell u_{i}, u_{i}\right) \leq \lambda_{i}\left(\omega u_{i}, u_{i}\right)+$ $\delta\left(u_{i}, u_{i}\right)$, whence for some constant $c$ sufficiently large,

$$
\left\|u_{i}\right\|_{H^{1,2}(\Omega)}^{2} \leq\left((\ell+c) u_{i}, u_{i}\right) \leq c+\delta .
$$


By the Sobolev-Kondrachoff compactness theorem [7, p. 167], without loss of generality we conclude that there is a function $u \in \stackrel{\circ}{H}^{1,2}(\Omega)$ such that $u_{i} \rightarrow u$ strongly in $L^{2}(\Omega)$ and weakly in $\stackrel{\circ}{H}^{1,2}(\Omega)$. Let $\varphi \in C_{0}^{\infty}\left(\Omega^{\prime \prime}\right)$. We observe $\left(\sum D_{j} u_{i}, D_{j} \varphi\right)+\left(q u_{i}, \varphi\right)=\left(\sum D_{j} u_{i}, D_{j} \varphi\right)+\left(q u_{i}-\lambda_{i} \omega u_{i}, \varphi\right)=k_{1}\left(\lambda_{i}\right)\left(u_{i}, \varphi\right)$.

By the continuity of the inner product in the various norms,

$$
\left(\sum D_{j} u, D_{j} \varphi\right)+(q u, \varphi)=\delta(u, \varphi) \text {. }
$$

It follows that $\ell u=\delta u$ in $\Omega^{\prime \prime}$. Next,

$$
\lim _{i \rightarrow \infty}\left[\left(\sum D_{j} u_{i}, D_{j} u_{i}\right)+\left(q u_{i}, u_{i}\right)-\left(k\left(\lambda_{i}\right) u_{i}, u_{i}\right)\right]=\lim _{i \rightarrow \infty} \lambda_{i}\left(\omega u_{i}, u_{i}\right) .
$$

Since the left hand side of $(4)$ is bounded and $\left(\omega u_{i}, u_{i}\right) \rightarrow(\omega u, u)$, we obtain $(\omega u, u)=0$ i.e. $u \equiv 0$ in $\Omega^{\prime}$, whence $u \in \stackrel{\circ}{H^{1,2}}\left(\Omega^{\prime \prime}\right)$ by Part (a). It follows that $\delta$ is an eigenvalue of $\ell$ in $\Omega^{\prime \prime}$, but since $\delta_{1} \geq \delta$ we conclude $\delta=\delta_{1}$. Suppose now the result has been established for $k_{j}, j=1, \ldots, p$ and consider $k_{p+1}(\lambda)$. Again by the Courant min.max., we observe that $\delta_{p+1} \geq k_{p+1}(\lambda)$ for any $\lambda$. On the other hand, $k_{p+1} \geq k_{p}$ and $\lim _{\lambda \rightarrow-\infty} k_{p}=\delta_{p}$ by assumption. The earlier procedures apply with no change, and we conclude that $\lim _{\lambda \rightarrow-\infty} k_{p+1}=\xi$ is an eigenvalue of (3) with $\delta_{p} \leq \xi \leq \delta_{p+1}$. But by the continuity of the inner products, $\delta_{p} \neq \xi$ as the eigenvectors in the subsequences corresponding to $k_{p}(\lambda), k_{p+1}(\lambda)$ in $\left(1^{\prime}\right)$ can be chosen orthogonal. It follows that $\xi=\delta_{p+1}$ and the result is shown.

Since Parts (d), (e) are immediate from Parts (a)-(c) once we observe that $k_{j}(\lambda) \rightarrow-\infty$ as $\lambda \rightarrow+\infty$ by the Courant min.max., we next show:

(f) If $\sum_{i=1}^{p} c_{i} u_{i} \equiv 0$ in $\Omega^{\prime}$, then without loss of generality assume $c_{i} \neq 0$ for $i=1, \ldots, p$, and that, by combining terms, $k_{i}(\lambda) \neq k_{j}(\lambda)$ if $i \neq j$. Note $(\ell-\lambda \omega)\left(\sum_{i=1}^{p} c_{i} u_{i}\right)=\sum_{i=1}^{p} k_{i}(\lambda) c_{i} u_{i} \equiv 0$ in $\Omega^{\prime}$. We conclude $\sum_{i=1}^{p-1} d_{i} u_{i}=0$ in $\Omega^{\prime}$ for some $d_{i} \neq 0$. By induction, $u_{1} \equiv 0$ in $\Omega^{\prime}$ whence $u_{1} \equiv 0$ in $\Omega$ by unique continuation. Since eigenvectors corresponding to the same (higher multiplicity) eigenvalue are chosen orthogonal, this is a contradiction.

Theorem 1 (c) can be compared with the asymptotic results of [3, Lemmae $4.1,4.2$.]. Note that in our case $\left(k_{j}(\lambda) / \lambda\right) \rightarrow 0$ as $\lambda \rightarrow-\infty$ for any chosen $j$.

Theorem 2. Let

$$
\begin{aligned}
& N_{1}=\#\left\{\lambda_{j} \mid \lambda_{j} \text { is a negative eigenvalue of }(1)\right\} ; \\
& N_{2}=\#\left\{\mu_{j} \mid \mu_{j} \text { is a negative eigenvalue of }(2)\right\} \\
& N_{3}=\#\left\{\delta_{j} \mid \delta_{j} \text { is a nonpositive eigenvalue of }(3)\right\} .
\end{aligned}
$$

Then $N_{2} \geq N_{3}$, and $N_{1}=N_{2}-N_{3}$.

Proof. That $N_{2} \geq N_{3}$ is an immediate consequence of the monotonicity of eigenvalues as domain functionals, since $\Omega^{\prime \prime} \subset \Omega$. To show that $N_{1}=N_{2}-N_{3}$ 
observe that Theorem 1 implies that $k_{j}(\lambda)<0$ for any finite $\lambda$ and any $j \leq N_{3}$, while there exists a sufficiently negative $\lambda_{j}^{*}$ such that $k_{j}\left(\lambda_{j}^{*}\right)>0$ and $k_{j}(0)<0$, $j=N_{3}+1, \ldots, N_{2}$. We conclude that $k_{j}(\lambda)=0$ has exactly one negative root for $j=N_{3}+1, \ldots, N_{2}$, whence (1) has $N_{2}-N_{3}$ negative eigenvalues.

We observe that $N_{1}$ depends only on $\Omega^{\prime \prime}$ and not on the specific values of $\omega$. Observe also that if $n=1$ (i.e. in the ordinary case) the number of zeros of the $j$ th eigenfunction of (1) is $N_{3}+j-1$, again a number independent of the specific values of $\omega$ on $\Omega^{\prime \prime}$.

We continue by observing the following min.max. Theorem for the eigenvalues $\left\{\lambda_{j}\right\}_{i=1}^{\infty}$ of (1). We define $\left\{\lambda_{j}\right\}_{j=1}^{\infty}$ as follows: Observe that $k_{j}(\lambda)=0$ has no roots for $j \leq N_{3}$ while it has exactly one for $j>N_{3}$. We define $\lambda_{j}$ to be the root of $k_{N_{3+j}}(\lambda)=0$ for $j=1, \ldots, \infty$.

Theorem 3. Let $S$ denote any subspace of $L^{2}(\Omega), S=\left\{u_{1}, \ldots, u_{p}\right\}$. Then

$$
\max _{S}\left[\inf _{\substack{u \in H^{1,2}(\Omega) \\
(u, S)=0 \\
(\omega u, u)>0}} \frac{(\ell u, u)}{(\omega u, u)}\right]=\left\{\begin{array}{ll}
-\infty & \left(p<N_{3}\right), \\
\lambda_{j} & \left(p=N_{3}+j-1\right) .
\end{array}\right]
$$

Proof. By definition, $k_{N_{3+j}}\left(\lambda_{j}\right)=0$. We select $S$ to be the space spanned by the eigenfunctions $\left\{u_{1}, \ldots, u_{p}\right\}$ of $\left(1^{\prime}\right)$ for $\lambda=\lambda_{j}$ and $k_{i}\left(\lambda_{j}\right) \leq k_{N_{3}+j}\left(\lambda_{j}\right)$, $i=1, \ldots, p$. If $u$ is the eigenvector of $\left(1^{\prime}\right)$ corresponding to $k_{N_{3}+j}\left(\lambda_{j}\right)$, we note that for $\varphi \in S^{\perp} \cap \stackrel{\circ}{H}^{1,2}$,

$$
0=k_{N_{3}+j}\left(\lambda_{j}\right)(\varphi, \varphi) \leq(\ell \varphi, \varphi)-\lambda_{j}(\omega \varphi, \varphi)
$$

with equality holding for $u$. Observe also that $(\omega u, u)>0$ by unique continuation. We conclude that

$$
\lambda_{j}=\inf _{\substack{(\varphi, S)=0 \\(\omega \varphi, \varphi)>0}} \frac{(\ell \varphi, \varphi)}{(\omega \varphi, \varphi)},
$$

with the infimum achieved by $u$. On the other hand, if $S$ is any given space, $S=\left\{\xi_{1}, \ldots, \xi_{p}\right\}$, let $u_{1}, \ldots, u_{p+1}$ be once again the first $p+1$ eigenfunctions of $\left(1^{\prime}\right)$. Choose $\left\{c_{i}\right\}$ such that $v=\sum_{i=1}^{p+1} c_{i} u_{i} \in S^{\perp}$. Observe that $(\omega v, v)>0$ by Theorem $1(\mathrm{f})$, and that $(\ell v, v)-\lambda_{j}(\omega v, v) \leq k_{N_{3}+j}\left(\lambda_{j}\right)(v, v)=0$. This implies

$$
\lambda_{j} \geq \inf _{\substack{v \in S^{\perp} \\(\omega v, v)>0}}\left[\frac{(\ell v, v)}{(\omega v, v)}\right] .
$$

Finally, suppose $p<N_{3}$. Observe that $k_{i}(\lambda)<0$ for all $\lambda$ and $i=1, \ldots, N_{3}$. Given any subspace $S=\left\{u_{1}, \ldots, u_{p}\right\}$ and $\tau \in \mathbf{R}^{1}$ let $v=\sum_{i=1}^{N_{3}} c_{i} v_{i} \in S^{\perp}$ as 
before, where $\ell\left(v_{i}\right)-\tau \omega v_{i}=k_{i}(\tau) v_{i}$. We conclude

$$
\begin{aligned}
(\ell v, v) & =\sum_{i=1}^{N_{3}} k_{i}(\tau) c_{i}^{2}\left(v_{i}, v_{i}\right)+\tau(\omega v, v) \\
& \leq \tau(\omega v, v) .
\end{aligned}
$$

Again by unique continuation, $(\omega v, v)>0$, whence

$$
\frac{(\ell v, v)}{(\omega v, v)} \leq \tau .
$$

Since $\tau$ is arbitrary, it follows that

$$
\inf _{\substack{v \in S^{\perp} \\\left(\omega v^{\prime}, v^{\prime}\right)>0}} \frac{(\ell v, v)}{(\omega v, v)}=-\infty .
$$

Observe that the proof of Theorem 3 shows that the condition $(\omega u, u)>0$ may be replaced by $\mu(\{u(x) \neq 0\} \cap B)>0$ for any sphere $B$. Combining the above arguments we obtain the following modest extension of [6, Theorem 3 (iv)] for our regularity and boundary conditions.

Corollary 4. Suppose $n=1$ and let $M_{j}$ denote the number of zeros of the $j$ th eigenfunction of (1). Then $M_{j}=j+N_{3}-1$. In particular, $M_{1}=0$ if and only if $N_{3}=0$ if and only if $\ell+\tau \omega>0$ for some $\tau \in \mathbf{R}^{1}$.

Note also that the result of Theorem 3 for $\lambda_{1}$ is in agreement with that obtained in [6, Theorem 6] for the $n=1$ and Neumann case. The rest of Theorem 3 appears to be new even for $n=1$.

For completeness, we observe that there is a classical min.max. associated with problem (1). Indeed, we may assume $\ell^{-1}: L^{2}(\Omega) \rightarrow L^{2}(\Omega)$ is a compact self-adjoint map (if not, we need only consider, by Theorem $1,(\ell+\alpha \omega)^{-1}$ for some $\alpha$ ). Setting $T=\omega^{1 / 2} \ell^{-1} \omega^{1 / 2}$ and following [8, p. 257] we observe that $\ell u=\lambda \omega u$ if and only if $v=\lambda T v$ with $v=\omega^{1 / 2} u$. Observe that the singularity of $\omega^{-1 / 2}$ causes no problem here. Consequently, $1 / \lambda$ corresponds to a point in the nonzero spectrum of $T$ and a classical min.max. is immediate by the Spectral Theorem, see e.g. [12, p. 233], applied to $T$. For example, the least positive eigenvalue $\lambda_{1}$ is given by

$$
\frac{1}{\lambda_{1}}=\sup _{\xi \in L^{2}(\Omega)} \frac{(T \xi, \xi)}{(\xi, \xi)}
$$

Note that since this min.max. involves $\ell^{-1}$ and not the original map $\ell$, it is awkward to apply to pariial differential equations. This approach does, however, yield an elementary proof of the completeness of the eigenvectors $\left\{u_{i}\right\}$ of (1) in the $L^{2}$ space with weight $\omega$ (see [6]). Specifically, if $(\omega u, v)=0$ for all $u$ eigenfunctions of $(1)$, then $\left(\omega^{1 / 2} u, \omega^{1 / 2} v\right)=0$. That is, $\omega^{1 / 2} v$ is perpendicular to the nonzero spectrum of $T$. By the Spectral Theorem, $T\left(\omega^{1 / 2} v\right)=0$, 
whence setting $\ell^{-1}(\omega v)=z$ and noting that $\omega^{1 / 2} z \equiv 0, \ell z=\omega v$, we obtain $\omega v \equiv 0$ in $\Omega^{\prime}$ since $\ell$ is a local map. But $\omega v=0$ in $\Omega^{\prime \prime}$ by definition of $\omega$ whence $(\omega v, v)=0$.

Comparison results can be formulated on the basis of Theorem 3. Consider two equations

$$
\ell_{i} u=\lambda \omega_{i} u
$$

for $i=2,3$ with $\ell_{i}, \omega_{i}$ satisfying analogous conditions to $\ell, \omega$. Let $N_{3}^{i}, \lambda_{j}^{i}$ denote the analogue of $N_{3}, \lambda_{j}$ for $\ell_{i}$. In the case of definite operators, the standard comparison result, [4, p. 411], states that if $\ell_{3} \geq \ell_{2}$ and $\omega_{3} \leq \omega_{2}$ then $\lambda_{j}^{3} \geq \lambda_{j}^{2}$ for $j=1, \ldots$. In our case, the situation is more complicated and reversals of these classical inequalities are possible.

Theorem 5. Suppose $\left(\ell_{3} \varphi, \varphi\right) \geq\left(\ell_{2} \varphi, \varphi\right),\left(\omega_{3} \varphi, \varphi\right) \geq\left(\omega_{2} \varphi, \varphi\right)$ for $\varphi \in \stackrel{\circ}{H}^{1,2}(\Omega)$. Then $N_{3}^{3} \leq N_{3}^{2}$. If $N_{3}^{3}=N_{3}^{2}$ and $\lambda_{j}^{3}<0$ for some index $j$, then $\lambda_{j}^{3} \geq \lambda_{j}^{2}$.

Proof. Observe that $\ell_{3} \geq \ell_{2}$ implies that $N_{3}^{3} \leq N_{3}^{2}$ by definition. Suppose $N_{3}^{3}=N_{3}^{2}$ and $\lambda_{j}^{3}<0$ for some $j$. Given some subspace $S$ as in Theorem 3, we observe

$$
0>\lambda_{j}^{3} \geq \inf _{\substack{\varphi \in H^{1.2}(\Omega) \\ \varphi \in S^{\perp}}}\left[\frac{\left(\ell_{3} \varphi, \varphi\right)}{\left(\omega_{3} \varphi, \varphi\right)}\right] .
$$

It follows that for $\varphi \in S^{\perp}$ with $\mu(\{\varphi \neq 0\} \cap B)>0$ for all spheres $B$, we have $0>\left(\ell_{3} \varphi, \varphi\right) \geq\left(\ell_{2} \varphi, \varphi\right)$ and $0<\left(\omega_{2} \varphi, \varphi\right) \leq\left(\omega_{3} \varphi, \varphi\right)$. For such $\varphi$,

$$
\frac{\left(\ell_{3} \varphi, \varphi\right)}{\left(\omega_{3} \varphi, \varphi\right)} \geq \frac{\left(\ell_{2} \varphi, \varphi\right)}{\left(\omega_{2} \varphi, \varphi\right)}
$$

and the result follows from Theorem 3 since $N_{3}^{3}=N_{3}^{2}$.

We remark, heuristically, that if $N_{3}^{3}<N_{3}^{2}$ then the comparison result of Theorem 5 fails in general. Indeed by considering the $k_{j}(\lambda)$ curves, it is easy in such a case to construct examples where $\lambda_{1}^{3}<\lambda_{1}^{2}$ and other examples where $\lambda_{1}^{3}>\lambda_{1}^{2}$. We also remark that it is possible, in the same way, to construct examples for which the conditions of Theorem 5 hold and yet $\lambda_{j}^{3} \leq \lambda_{j}^{2}$ for $j$ large. One need only take $\ell_{3}=\ell_{2}$ and $\omega_{3}-\omega_{2}$ sufficiently large. These simple remarks indicate that while comparison theorems are formally possible for $\left(5_{i}\right)$, more conditions than just $\ell_{3} \geq \ell_{2}$ and $\omega_{3} \geq \omega_{2}$ (or their opposites) are always needed.

\section{Applications}

We continue the paper with an application and an illustrative example. We focus our attention on estimating the negative spectrum (if any) of equation (1). 
Theorem 6. Assume $\mu_{1} \leq \ldots \mu_{N_{2}}<0<\mu_{N_{2}+1}$ be the first eigenvalues of (2) with associated normalized (in $L^{2}$ ) eigenvectors $\left\{u_{i}\right\}_{1}^{N_{2}+1}$. Set $\tilde{\omega}=\omega /\|\omega\|_{\infty}$, $\tau=$ least eigenvalue of the matrix $\left(\left(\tilde{\omega} u_{i}, u_{j}\right)\right)_{N_{3}+1}^{N_{2}}$ and assume

$$
\tau>\left(-\mu_{N_{3}+1}\right) /\left(\mu_{N_{2}+1}\right) .
$$

Then the negative spectrum of (1) is contained in the interval

$$
\frac{1}{\|\omega\|_{\infty}}\left[\frac{\mu_{N_{3}+1}}{\left[\tau^{1 / 2}-\left(-\frac{\mu_{N_{3}+1}}{\mu_{N_{2}+1}}\right)^{1 / 2}\right]^{2}}, \mu_{N_{2}}\right] .
$$

Proof. By Theorems 2, 3 it follows that if (1) has negative spectrum then $N_{3}<$ $N_{2}$ and the first eigenvalue $\lambda_{1}$ of (1) satisfies

$$
\lambda_{1} \geq \inf _{\substack{\varphi \in S^{\perp} \\(\omega \varphi, \varphi)>0}}\left[\frac{(\ell \varphi, \varphi)}{(\omega \varphi, \varphi)}\right]
$$

where $S$ is now explicitly chosen by $S=\left\{u_{i}\right\}_{i=1}^{N_{3}}$, with $u_{i}$ normalized (in $L^{2}$ ) eigenfunctions of (2). If $\varphi \in S^{\perp}$, we may express $\varphi$ as $\varphi=\sum_{N_{3}+1}^{N_{2}} c_{i} u_{i}+v$ with $(\ell v, v) \geq \mu_{N_{2}+1}(v, v)$. Since $(\omega \varphi, \varphi)>0$, we may also without loss of generality assume that $(\ell \varphi, \varphi) \leq 0$, i.e. $\sum_{N_{3}+1}^{N_{2}} c_{i}^{2} \mu_{i}+(\ell v, v) \leq 0$. That is,

$$
\mu_{N_{2}+1}(v, v) \leq(\ell v, v) \leq-(\ell u, u),
$$

where we set $u=\sum_{N_{3}+1}^{N_{2}} c_{i} u_{i}$. This yields

$$
\begin{aligned}
(\omega \varphi, \varphi) & =(\omega u, u)+2(\omega u, v)+(\omega v, v) \\
& \geq\left[(\omega u, u)^{1 / 2}-(\omega v, v)^{1 / 2}\right]^{2}
\end{aligned}
$$

by Holder's Inequality. It follows that

$$
(\omega \varphi, \varphi) \geq\|\omega\|_{\infty}\left[(\tilde{\omega} u, u)^{1 / 2}-\left(\frac{-1}{\mu_{N_{2}+1}}(\ell u, u)\right)^{1 / 2}\right]^{2} .
$$

We conclude,

$$
\frac{(\ell \varphi, \varphi)}{(\omega \varphi, \varphi)} \geq \frac{\sum_{N_{3}+1}^{N_{2}} \mu_{i} c_{i}^{2}}{\|\omega\|_{\infty}\left[\left(\tau^{1 / 2}\right)-\left(-\frac{\mu_{N_{3}+1}}{\mu_{N_{2}+1}}\right)^{1 / 2}\right]^{2} \sum c_{i}^{2}} .
$$

This yields

$$
\lambda_{1} \geq \frac{\mu_{N_{3}+1}}{\|\omega\|_{\infty}\left[\left(\tau^{1 / 2}\right)-\left(-\frac{\mu_{N_{3}+1}}{\mu_{N_{2}+1}}\right)^{1 / 2}\right]^{2}} .
$$


Finally, observe that by Theorem $1, k_{j}^{\prime} \geq-\|\omega\|_{\infty}$ a.e. whence

$$
k_{N_{2}}\left(\lambda_{\left(N_{2}-N_{3}\right)}\right)+\int_{\lambda_{\left(N_{2}-N_{3}\right)}}^{0} k_{N_{2}}^{\prime}=\mu_{N_{2}}
$$

and we conclude

$$
\lambda_{\left(N_{2}-N_{3}\right)} \leq \frac{\mu_{N_{2}}}{\|\omega\|_{\infty}} .
$$

Since $\lambda_{\left(N_{2}-N_{3}\right)}$ is the largest negative eigenvalue of $(1)$, the proof is complete.

The advantage of Theorem 6 is that the eigenfunctions and eigenvalues needed for the estimate are all from equation (2) and hence may be explicitly known. Inequality (6), however, does present a severe restriction. Indeed, it may appear at first sight that Theorem 6 is vacuous because of this inequality. To show that this is not the case, we conclude with the following example. For simplicity, we consider an ordinary differential equation but analogous examples are easily constructed in higher dimensions.

Corollary 7. Let $n=1, \Omega=(0,2 \pi), \Omega^{\prime}=(0, \pi)$, and assume $\ell u=-u^{\prime \prime}-k u$ with $k=1+\varepsilon$ and $0<\varepsilon<5 / 4$. Then $\ell u=\lambda \omega u$ has exactly one negative eigenvalue, $\lambda_{1}$, and if

$$
\tau=\frac{1}{\pi} \int_{0}^{\pi} \tilde{\omega} \sin ^{2}(x)>\frac{\varepsilon}{(5 / 4-\varepsilon)} \triangleq \beta,
$$

then

$$
\lambda_{1} \in \frac{1}{\|\omega\|_{\infty}}\left[-\frac{\varepsilon}{\left(\tau^{1 / 2}-\beta^{1 / 2}\right)^{2}},-\varepsilon\right] .
$$

Proof. That $\ell u=\lambda \omega u$ has only one negative eigenvalue is immediate from Theorem 2, since the spectrum of equations (2), (3) is explicitly known. We thus have $N_{3}+1=N_{2}=2$ and $\mu_{N_{2}}=-\varepsilon$. Note that here $\tau=\frac{1}{\pi} \int_{0}^{\pi} \tilde{\omega} \sin ^{2}(x)$; while $\left(-\mu_{N_{3}+1}\right) /\left(\mu_{N_{2}+1}\right)=\beta$.

Despite its special nature, we are not aware of other results along the lines of Corollary 7. Observe that the maximum $\varepsilon$ will usually have to be chosen depending on $\tilde{\omega}$. If for example, $\tilde{\omega}=\sin x$ in $(0, \pi)$ then $\tau=4 / 3 \pi$ and, consequently, $\varepsilon<5 /(3 \pi+4)$. Conversely, if $\varepsilon$ is chosen first then $\tau$ (i.e. $\tilde{\omega}$ ) will need be chosen sufficiently large in order for Corollary 7 to be applicable.

\section{CONCLUDING REMARKS}

We end the paper with the following heuristic remarks on the procedures to be employed in slightly more general cases.

Suppose first that $\Omega^{\prime \prime}$ is not connected, but, instead, $\Omega^{\prime \prime}=\bigcup_{1}^{p} O_{i}$ with each $O_{i}$ a smooth domain and $O_{i} \cap O_{j}=\varnothing$ if $i \neq j$. The only modification needed in this situation is based on the observation that the spectrum of $(3)$ is now the union of the spectra for each $O_{i}$. In particular, (3) will have $p$ nonnegative nontrivial eigenvectors. 
Secondly, assume that $u \in V$ rather than $u \in \stackrel{\circ}{H}^{1,2}(\Omega)$. This is the case to which we alluded in the Introduction. In this situation, the boundary conditions in $\left(1^{\prime}\right)$, (2) become $u \in V, v \in V$ respectively. However, the boundary condition in (3) are mixed. For example if $V=H^{1,2}(\Omega)$ then they are: $u=0$ on $\partial \Omega^{\prime}, \partial u / \partial n=0$ on $\partial \Omega$ (in a generalized sense). Note that the condition $u=0$ on $\partial \Omega^{\prime}$ still guarantees the monotonicity of eigenvalues as domain functionals.

In both of the above cases, results can be obtained by elementary variations in the procedures we followed.

\section{REFERENCES}

1. W. Allegretto and A. Mingarelli, On the non-existence of positive solutions for a Schrödinger equation with an indefinite weight function, C.R. Math. Rep. Acad. Sci. Canada 8 (1986), 69-73.

2. __ Boundary problems of the second order with an indefinite weight function, preprint.

3. P. Binding and P. Browne, Spectral properties of two parameter eigenvalue problems II, Proc. Royal Soc. Edinb. 106A(1987), 39-51.

4. R. Courant and D. Hilbert, Methods of mathematical physics, Vol. I, Interscience, New York, 1953.

5. W. N. Everitt, M. Kwong and A. Zettl, Oscillation of eigenfunctions of weighted regular SturmLiouville problems, J. London Math. Soc. 27(1983), 106-120.

6. __ Differential operators and quadratic inequalities with a degenerate weight, J. Math. Anal. Appl. 98 (1984), 378-399.

7. D. Gilbarg and N. S. Trudinger, Elliptic partial differential equations of second order, 2nd Edition, Springer, Berlin/New York, 1983.

8. I. C. Gohberg and M. G. Krein, Theory and applications of Volterra operators in Hilbert space, Trans. of Math. Mono., Vol. 24, American Mathematical Society, 1970.

9. E. L. Ince, Ordinary differential equations, Dover, New York, 1956.

10. M. Reed and B. Simon, Methods of modern mathematical physics, Vol. IV, Academic Press, New York, 1978.

11. F. Rellich, Perturbation theory of eigenvalue problems, Gordon and Breach, New York, 1969.

12. F. Reisz and B. Sz.-Nagy, Functional analysis, Ungar Publishing, New York, 1955.

13. S. L. Sobolev, Applications of functional analysis in mathematical physics, Trans. of Math. Mono., Vol. 7, American Mathematical Society, 1963.

14. R. Vyborny, Continuous dependence of eigenvalues on the domain, Lecture Sec. No. 42, Institute for Fluid Dynamics and Applied Mathematics, Univ. of Maryland, 1964.

Department of Mathematics, University of Alberta, Edmonton, Alberta, Canada T6G 2G1 